\title{
RECONSTRUCTION OF THE ${ }^{14} \mathrm{C}$ PRODUCTION RATE FROM MEASURED RELATIVE ABUNDANCE
}

\author{
Ilya G Usoskin \\ Sodankylä Geophysical Observatory (Oulu unit), P.O. Box 3000, FIN-90014 University of Oulu, Finland. \\ Email: Ilya.Usoskin@oulu.fi. \\ Bernd Kromer \\ Heidelberger Akademie der Wissenschaften, Institut für Umweltphysik, INF 229, D-69120 Heidelberg, Germany. \\ Email: Bernd.Kromer@iup.uni-heidelberg.de.
}

\begin{abstract}
A new method is presented for the reconstruction of the radiocarbon production rate from the measured relative abundance of $\Delta^{14} \mathrm{C}$. The method treats the carbon cycle as a linear Fourier filter and thus allows for the correct and unambiguous inversion of the carbon cycle. The ${ }^{14} \mathrm{C}$ production rate, as reconstructed by the Fourier filter method, agrees with the results obtained by the traditional iteration method. Since the 2 methods use completely different approaches, this verifies the validity of the reconstruction. The composite series is presented, based on both methods and their systematic uncertainties.
\end{abstract}

\section{INTRODUCTION}

Information on the cosmic ray flux in the past is of great importance for solar and heliospheric physics as well as for the study of solar-terrestrial relations on long time scales (e.g. Stuiver et al. 1991; Bond et al. 2001). For the time before the era of direct observations of sunspots and related phenomena like aurorae, only cosmogenic isotopes such as ${ }^{14} \mathrm{C},{ }^{10} \mathrm{Be}$, and ${ }^{36} \mathrm{Cl}$ can provide a quantitative reconstruction of the cosmic ray flux. Although the radiocarbon production rate is directly proportional to the flux of cosmic rays with energy around a few $\mathrm{GeV} /$ nucleon impinging on Earth (Castagnoli and Lal 1980; Alanko et al. 2003), the direct comparison of the relative ${ }^{14} \mathrm{C}$ abundance in tree rings $\left(\Delta^{14} \mathrm{C}\right)$ with the cosmic ray flux is not straightforward. Several processes disturb the cosmic ray signal in the relative ${ }^{14} \mathrm{C}$ concentration in natural archives. One process is related to the long-term changes of the geomagnetic field which shields Earth from low-energy cosmic rays. Using independent paleomagnetic data, one can take this effect into account. The most important process distorting the cosmic signal in $\Delta^{14} \mathrm{C}$ is the complex carbon cycle, which effectively leads to attenuation and variable time delay of the cosmic ray signal. While the carbon cycle has been successfully modeled and the measured $\Delta^{14} \mathrm{C}$ series can be precisely calculated if the ${ }^{14} \mathrm{C}$ production rate is known from other sources (Siegenthaler et al. 1980; Bard et al. 1997), an inversion of this process (i.e. reconstruction of the production rate from the measured concentration) is not straightforward. Some attempts have been made to solve this problem. For example, Stuiver and Quay (1980) made an assumption on the ${ }^{14} \mathrm{C}$ production rate and then fitted the calculated $\Delta^{14} \mathrm{C}$ variations to the actual measurements. Kocharov et al. (1977) suggested to extract a few basic periodicities from the original signal and to treat them separately, but they did not reconstruct the production rate. Kocharov et al. (1983) solved, under simplifying assumptions, a system of linear differential equations describing a 5-reservoir carbon cycle model to determine the ${ }^{14} \mathrm{C}$ production rate. However, their results appeared to be dependent on the assumption of the cosmic ray spectrum. In this paper, we suggest a new method to reconstruct the ${ }^{14} \mathrm{C}$ production rate from the measured $\Delta{ }^{14} \mathrm{C}$ which treats the carbon cycle as a Fourier filter, and compare it to the earlier method based upon an iteration approach using the carbon cycle model. 


\section{CARBON CYCLE AND THE OESCHGER-SIEGENTHALER BOX MODEL}

The carbon cycle in the interrelated system atmosphere-biosphere-ocean is usually described using a box model where the carbon cycle is represented by fluxes between different carbon reservoirs and mixing within the ocean reservoir(s). For the purpose of studying ${ }^{14} \mathrm{C}$, production and radioactive decay are also included in box models. Free parameters in the Oeschger-Siegenthaler type box model (Oeschger et al. 1974; Siegenthaler et al. 1980) are the ${ }^{14} \mathrm{C}$ production rate $Q$, the air-sea exchange rate (expressed as turnover rate $k$ ), and the vertical eddy diffusion coefficient $K$, which combines ocean ventilation into a single parameter. Starting from the original representation (Oeschger et al. 1974), a variety of box models have been developed which take into account subdivisions of the ocean reservoir and direct exchange between the deep ocean and the atmosphere at high latitudes. These models have been run in prognostic mode for which the time series of parameter variations are prescribed, such as a reduction in ocean ventilation or sea-ice cover, and the response of the atmospheric $\Delta^{14} \mathrm{C}$ level is obtained as output of the model. Obviously, box models of higher complexity allow more realistic scenarios to be tested, but they require a prescription of a larger number of model parameters. However, for the inversion of the model, i.e. to evaluate the ${ }^{14} \mathrm{C}$ production rate $Q$ from the variations of $\Delta^{14} \mathrm{C}$ to first order, only the production rate is considered variable, while the gas-exchange rate and ocean mixing are kept constant. Hence, any subdivision of reservoirs or processes would not make sense and the simplest version of a carbon box model is sufficient for this goal. In order to reconstruct the ${ }^{14} \mathrm{C}$ production rate, we ran a version of the OeschgerSiegenthaler box model (Born 1994) using the pre-industrial steady-state values $\left(K=4200 \mathrm{~m}^{2} \mathrm{yr}^{-1}\right.$, $k=1 / 6.9 \mathrm{yr}$ ) and a steady-state ocean-depth profile of ${ }^{14} \mathrm{C}$ based on $\Delta{ }^{14} \mathrm{C}=135 \%$ in the top ocean box at the time of the start of the run $(11.445 \mathrm{BP})$. The ${ }^{14} \mathrm{C}$ production rate has been calculated iteratively in each time step $(0.01 \mathrm{yr})$ as the difference between the observed $\Delta^{14} \mathrm{C}$ measured in absolutely dated tree rings (interpolated from the decadal data of IntCal98, Stuiver et al. 1998) and the model-calculated $\Delta^{14} \mathrm{C}$. The production rate is obtained relative to the modern production rate of 2.2 atoms $\mathrm{cm}^{-2} \mathrm{~s}^{-1}$, although this value may be higher than the steady-state production calculated from the ${ }^{14} \mathrm{C}$ inventory in the model reservoirs (see, e.g., Goslar 2001 and references therein).

\section{CARBON CYCLE AS A FOURIER FILTER}

All carbon cycle models consist of a system of linear differential equations describing the carbon exchange between several reservoirs. Consequently, such a process works as a linear Fourier filteri.e. it does not change the shape of a propagating harmonic signal so that a sine wave is amplified/ attenuated and phase-shifted, but its frequency remains the same when propagating through this process either in forward or in backward direction. In such circumstances, the carbon cycle can be considered as a Fourier filter process $G$. Let us denote the functions describing the ${ }^{14} \mathrm{C}$ production rate as $Q$ and $q$ and those for the $\Delta^{14} \mathrm{C}$ abundance as $D$ and $d$, where capital and small letters denote the functions in the time $(t)$ and frequency $(f)$ domains, respectively. We denote the direct and backward Fourier transform operations as $F$ and $F^{-1}$ so that

$$
\begin{gathered}
q(f)=F[Q(t)] \equiv \int_{-\infty}^{\infty} Q(t) e^{2 \pi i f t} d t \\
Q(t)=F^{-1}[q(f)] \equiv \int_{-\infty}^{\infty} q(f) e^{-2 \pi i f t} d f
\end{gathered}
$$

Using this formalism, the carbon cycle can be formally presented as follows:

$$
Q(t) \stackrel{F}{\rightarrow} q(f) \stackrel{G}{\rightarrow} d(f) \stackrel{F^{-1}}{\rightarrow} D(t)
$$



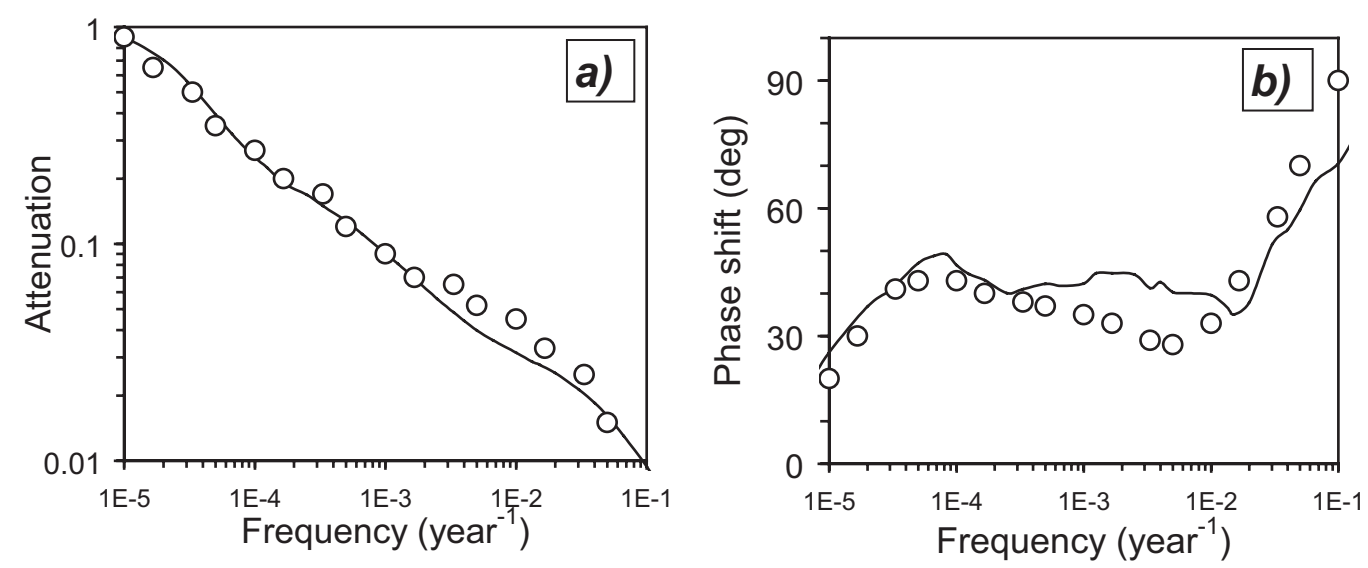

Figure 1 The frequency characteristics of the attenuation and phase-shift coefficients in the carbon atmospheric transport model: line - according to the Oeschger-Siegenthaler box model (Born 1994) used here, and open dots - according to the PANDORA model (e.g. Bard et al. 1997).

First, the original signal (the production rate, $Q[t]$ ) is transformed to the Fourier series $q(f)$, then the filter $G$ describing the carbon transport model is applied to obtain the Fourier representation $d(f)$ of the $\Delta^{14} \mathrm{C}$ series. Finally, $d(f)$ is transformed back to the time series $D(t)$ of $\Delta{ }^{14} \mathrm{C}$. Since the filter $G$ is linear in the frequency domain, the chain (2) can be unambiguously inverted,

$$
Q(t) \stackrel{F^{-1}}{\rightarrow} q(f) \stackrel{G^{-1}}{\rightarrow} d(f) \stackrel{F}{\rightarrow} D(t)
$$

so that by decomposing the measured $\Delta^{14} \mathrm{C}$ series, passing it through the (inverted) filter and composing it again, one can obtain the time series of the ${ }^{14} \mathrm{C}$ production rate. Based upon the general approach described above, we now consider the details of the ${ }^{14} \mathrm{C}$ production rate reconstruction. In our calculations, we use the FFT method, substituting the integrals in Equation 1 by a finite sum,

$$
\begin{gathered}
Q_{k}=Q(k \Delta t)=\sum_{m=-N / 2}^{N /(2-1)} q_{m} e^{(2 \pi i m t) /(N \Delta t)} \\
q_{k}=\sum_{m=-N / 2}^{N /(2-1)} Q_{m} e^{[(-2) \pi i m k] / N}
\end{gathered}
$$

where $\Delta t$ is the sampling rate and $N$ is the number of the points in the series. The frequency domain representation is $q_{k} \equiv R\left(q_{k}\right) \exp \left[i \phi\left(q_{k}\right)\right]$, where $R\left(q_{k}\right)$ and $\phi\left(q_{k}\right)$ are the amplitude and phase of $q_{k}$, respectively. The frequency characteristics of amplification/attenuation and phase-shift coefficients, also called Bode plots (Bode 1945), completely define a process (Jenkins and Watts 1969). The Bode plots of the filter $G$ corresponding to the Oeschger-Siegenthaler box model (Born 1994) are shown in Figure 1 together with the frequency characteristics of the 12-box PANDORA model (e.g. Bard et al. 1997). Applying this filter $G$ to the series $q_{k}$, one can obtain the series $d_{k}=G\left(q_{k}\right)$ as follows:

$$
R\left(d_{k}\right)=A_{k} \times R\left(q_{k}\right), \phi\left(d_{k}\right)=\phi\left(q_{k}\right)+\phi_{k}
$$

where $A_{k}$ and $\phi_{k}$ are the attenuation coefficient and the phase shift for the $k^{\text {th }}$ frequency, respectively (see Figure 1). Inverting the filter, one can obtain the Fourier series $q_{k}=G^{-1}\left(d_{k}\right)$ from $d_{k}$ : 


$$
R\left(q_{k}\right)=R\left(d_{k}\right) / A_{k}, \phi\left(q_{k}\right)=\phi\left(d_{k}\right)-\phi_{k}
$$

The representations of the series $q(f)$ and $d(f)$ in the frequency domain are shown in Figure 2. By applying the inverse Fourier transform, we obtain the ${ }^{14} \mathrm{C}$ production rate $Q(t)$ from $q(f)$.

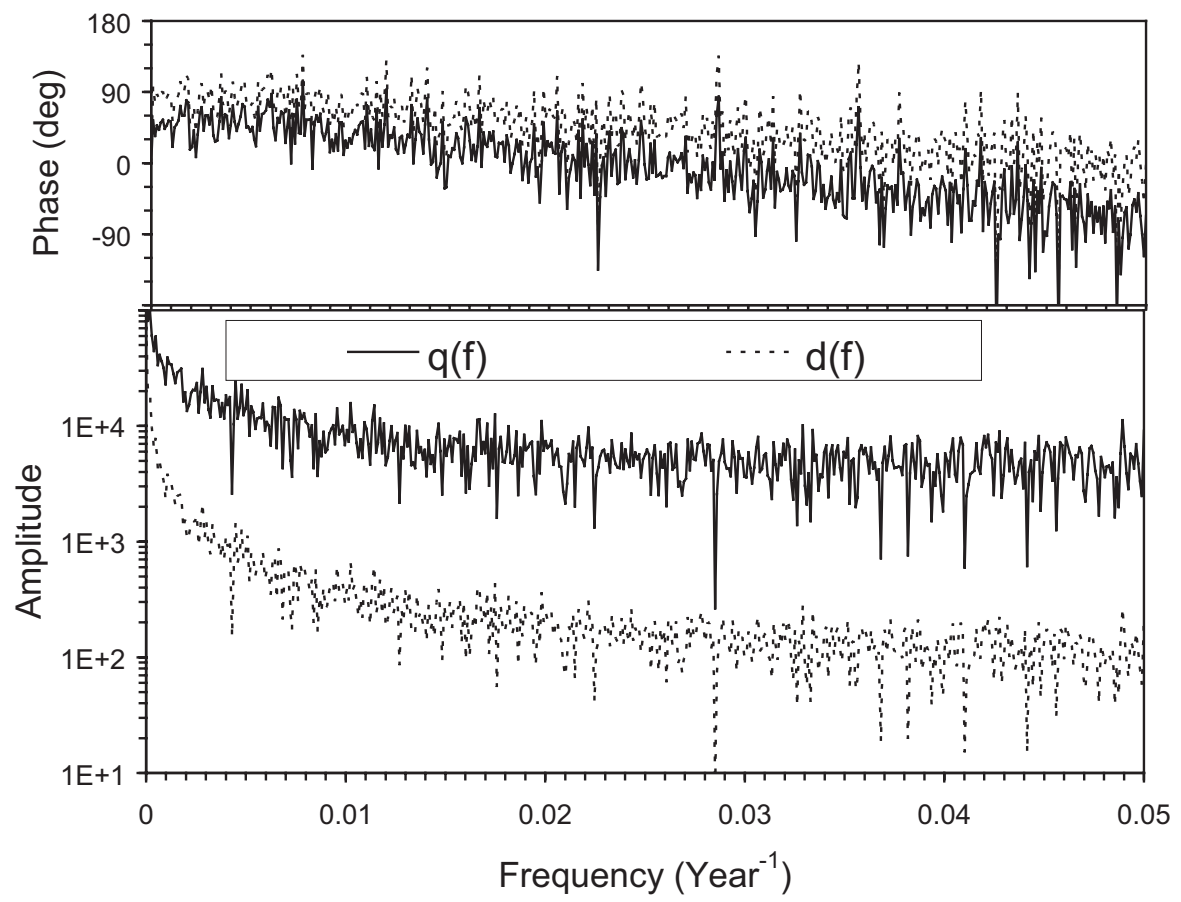

Figure 2 The representations of the production rate $q(f)$ (solid line) and the $\Delta^{14} \mathrm{C}$ series $d(f)$ (dotted line) in the frequency domain. The top and bottom panels depict the phase and amplitude, respectively.

The Fourier filter approach provides a useful tool to study cosmic ray flux in the past using the ${ }^{14} \mathrm{C}$ data. Once the frequency characteristics (amplification and phase-shift coefficients) of the carbon transport model are calculated, the method can be readily applied. However, this approach has some shortcomings. Its application is hardly possible for frequencies higher than $0.1 \mathrm{yr}^{-1}$ since the very strong attenuation coefficient in the high-frequency range results in amplification of the noise that is always present in the measured data due to, e.g., local fluctuations of the carbon deposition or due to measurement errors. Accordingly, the high-frequency part of the reconstructed series is dominated by the amplified noise. Another limitation of the method for high frequency is that it substitutes the real-time delay, $\tau_{k}$, in the carbon cycle for a frequency $f_{k}$ (corresponding to period $T_{k}$ ) with a phase shift $\phi_{k}=2 \pi \tau_{k} / T_{k}$. This is possible only if the time delay is shorter than half of the period in the signal $\left(\tau_{k}<T_{k} / 2\right)$, which is not correct for the high-frequency part of the model and leads to the saturation of the phase shift at $90^{\circ}(\pi / 2)$ for periods shorter than $10 \mathrm{yr}$ (see Figure 1). Therefore, this method cannot reconstruct annual data but requires sampling or low-pass smoothing of the data series with the frequency lower than $0.1 \mathrm{yr}^{-1}$. Also, the reconstructed data become uncertain near the beginning of the time series because the effective phase shift refers to the unavailable earlier data. This is equivalent to the uncertainties of the initial conditions in the iteration method. 


\section{RESULTS}

We have presented a reconstruction of the ${ }^{14} \mathrm{C}$ production rate from the relative abundance $\Delta^{14} \mathrm{C}$ measured in tree rings, with decadal resolution (Stuiver et al. 1998), using the 2 different approaches described above. Both methods yield similar results. An example of the 2 reconstructions of $Q$ is shown in Figure 3 for the last 2 millennia together with the measured $\Delta^{14} \mathrm{C}$. The 2 reconstructed series of the production rate nearly coincide with each other during this as well as during the entire 11,400-yr time intervals (Figure 4). Taking into account that the 2 reconstructions have been performed using very different methods - the iteration fitting procedure and the Fourier filter approach, both based upon the same description of the carbon cycle (Born 1994) - the present result verifies both methods for reconstruction of the ${ }^{14} \mathrm{C}$ production rate. We note that using the frequency characteristics of another carbon cycle model, the 12-box PANDORA model (Bard et al. 1997), leads to the very similar results. However, we would like to stress that both methods assume steadystate conditions at the beginning of the reconstruction and the subsequent $\Delta^{14} \mathrm{C}$ in the atmosphere defined solely by changes in the production rate. While variability in the carbon cycle is believed to be rather small during the Holocene and production changes are considered to dominate the decadalto century-scale $\Delta^{14} \mathrm{C}$ fluctuations, the assumption of the steady-state initial condition is possibly invalid for the first few millennia following deglaciation.

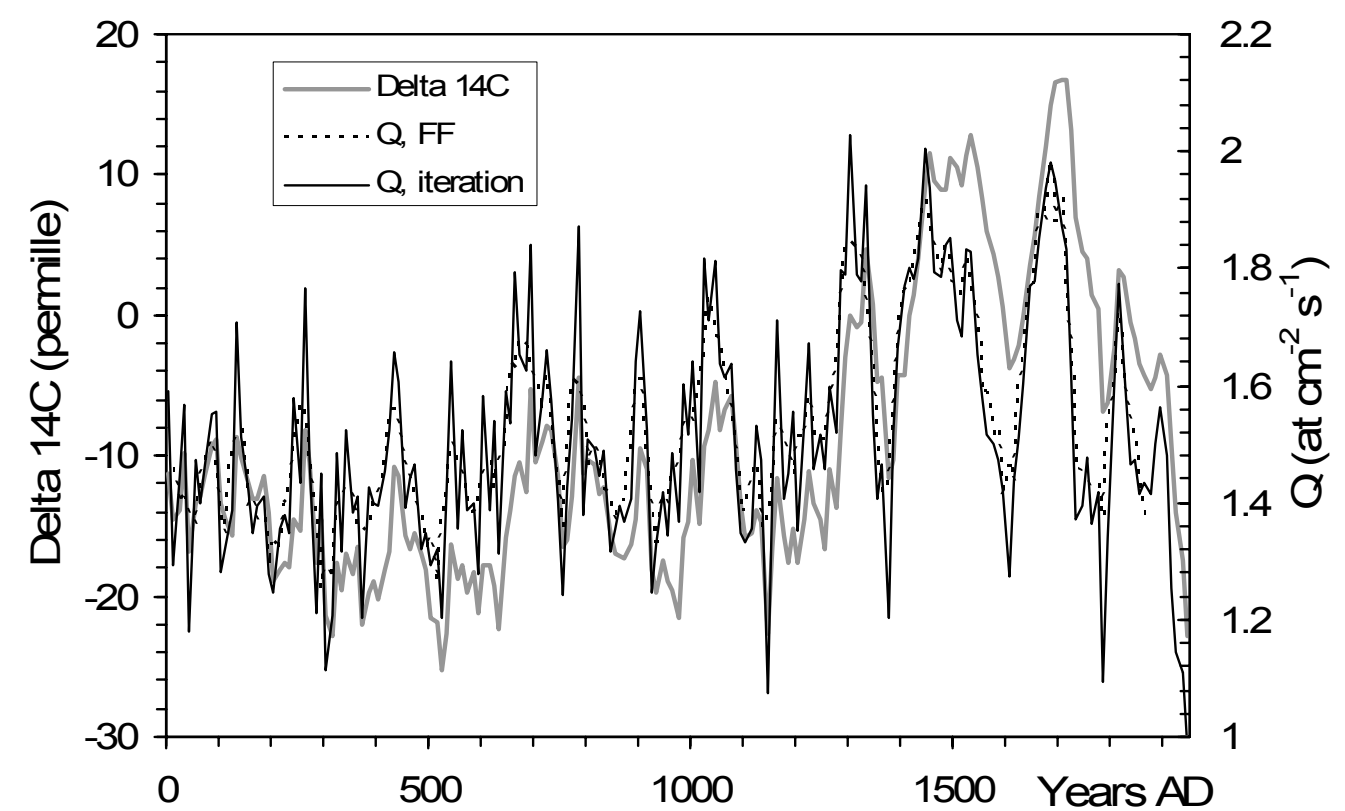

Figure 3 The ${ }^{14} \mathrm{C}$ profiles for the last 2 millennia. The measured decadal $\Delta{ }^{14} \mathrm{C}$ (Stuiver and Braziunas 1993) is shown by the gray curve (left axis). The reconstructed ${ }^{14} \mathrm{C}$ production rate $Q$ (right axis) is shown for the iteration method (solid bold curve) and for the Fourier filter (dashed curve) approach.

A composite ${ }^{14} \mathrm{C}$ production rate series, which is the average of the 2 reconstructed series, is given in the upper panel of Figure 5, while the lower panel shows the deviations between the individual and the average series, which serves as a measure of the model uncertainty. The standard deviation is 0.04 (atoms cm $\mathrm{s}^{-2} \mathrm{~s}^{-1}$ ). The mean deviation is zero, and only beyond 11,000 BP do the 2 series start diverging due to the uncertainties near the beginning of the series. Accordingly, our reconstructed ${ }^{14} \mathrm{C}$ production rate series is verified by the 2 very different techniques. 


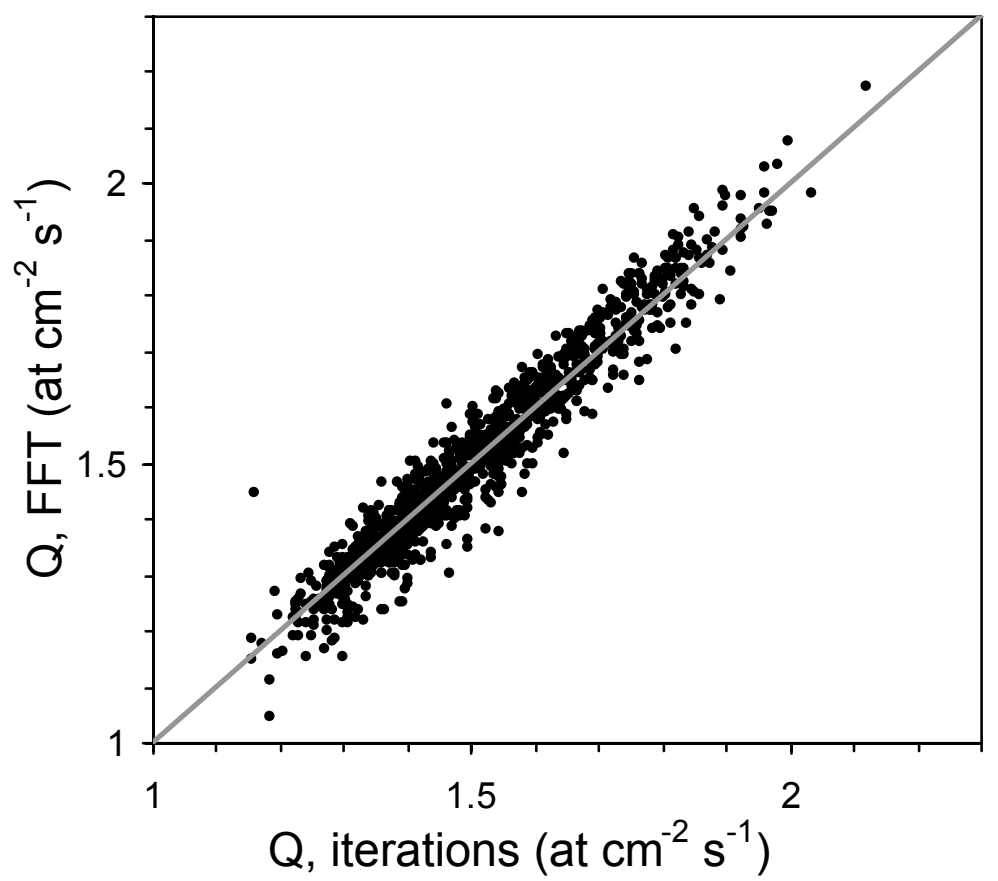

Figure 4 Scatter plot of the ${ }^{14} \mathrm{C}$ production rate $Q$ reconstructed since $11,400 \mathrm{BP}$ by the 2 different approaches: the iteration method and the Fourier filter.
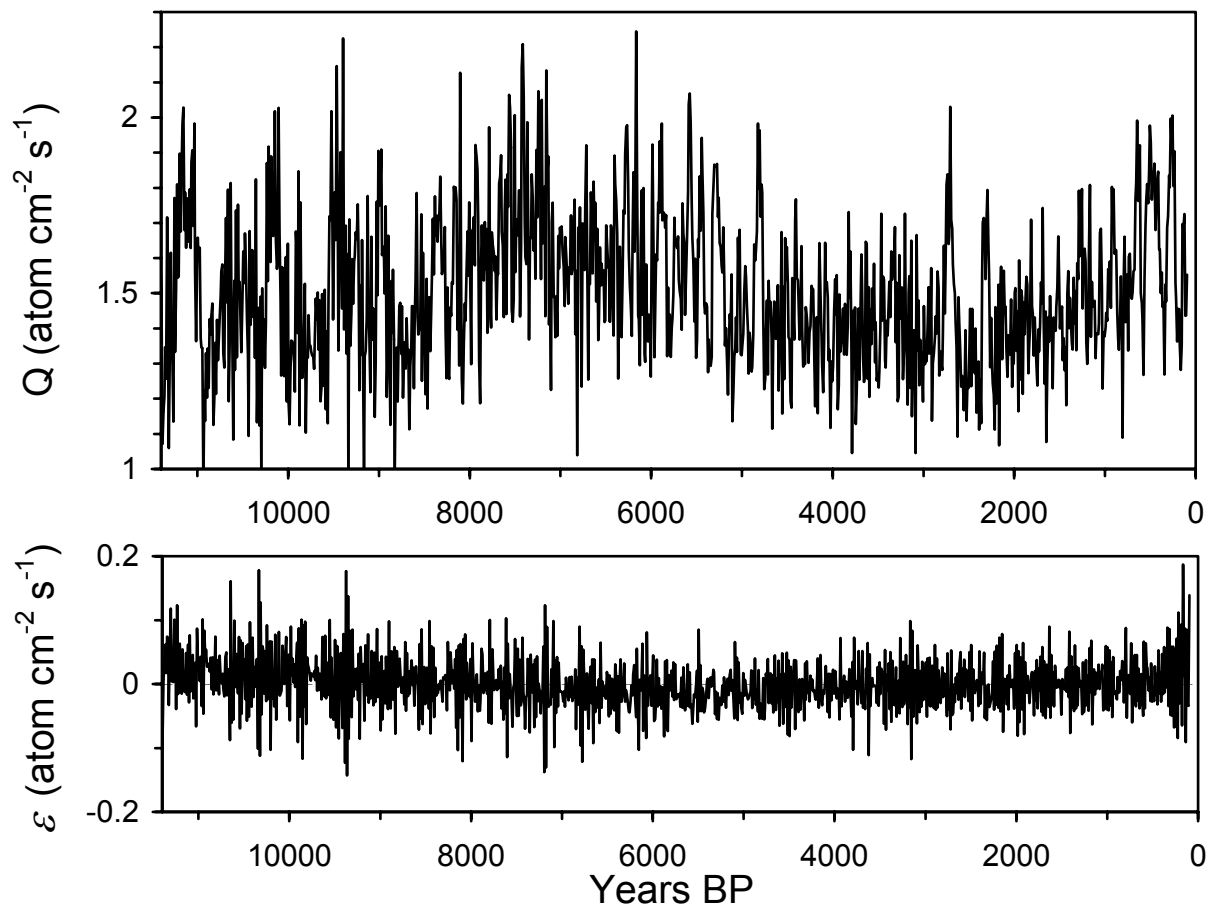

Figure 5 The ${ }^{14} \mathrm{C}$ production rate $Q$ composed as the average of the 2 reconstructions (upper panel) and the systematic errors of the production rate due to the difference in reconstruction methods (bottom panel). 


\section{CONCLUSION}

The composite series presented here of the ${ }^{14} \mathrm{C}$ production rate on the multi-millennium time scale has important implications for the long-term studies of cosmic rays, solar and heliosphere physics, solar-terrestrial relations, climate, etc. For example, it is common to check the consistency of the ${ }^{10} \mathrm{Be}$ and ${ }^{14} \mathrm{C}$ cosmogenic isotope data by assuming that the ${ }^{14} \mathrm{C}$ production rate is proportional to the ${ }^{10} \mathrm{Be}$ concentration in polar ice, calculating the expected $\Delta^{14} \mathrm{C}$ employing a direct carbon cycle model and comparing it to the measured $\Delta^{14} \mathrm{C}$ (e.g. Bard et al. 1997). The approach suggested here permits for a direct comparison of the ${ }^{14} \mathrm{C}$ production rate to ${ }^{10} \mathrm{Be}$ data and to study their common features and discrepancies.

\section{ACKNOWLEDGMENTS}

We thank Jürg Beer, Gennady Kovaltsov, Manfred Schüssler, and Sami Solanki for stimulating discussions and comments on the manuscript.

\section{REFERENCES}

Alanko K, Usoskin IG, Mursula K, Kovaltsov GA. 2003. Effective energy of Neutron Monitor. Proceedings of the 28th International Cosmic Ray Conference. Tokyo: Universal Academy Press. p 3901-4.

Bard E, Raisbek GM, Yiou F, Jouzel J. 1997. Solar modulation of cosmogenic nuclide production over the last millennium: comparison between ${ }^{14} \mathrm{C}$ and ${ }^{10} \mathrm{Be}$ records. Earth and Planetary Science Letters 150: 453-62.

Bode HW. 1945. Network Analysis and Feedback Amplifier Design. New York: Van Nostrand. 577 p.

Bond G, Kromer B, Beer J, Muscheler R, Evans M, Showers W, Hofmann S, Lotti-Bond R, Hajdas I, Bonani G. 2001. Persistent solar influence on North Atlantic surface circulation during the Holocene. Science 294:2130-6.

Born M. 1994. Optimierung eines Messsystems zur ${ }^{14} \mathrm{C}$ Aktivitätsbestimmung mit Proportionalzählrohren [PhD dissertation]. Heidelberg: University of Heidelberg. $81 \mathrm{p}$.

Castagnoli G, Lal D. 1980. Solar modulation effects in terrestrial production of carbon-14. Radiocarbon 22(2): 133-58.

Goslar T. 2001. Absolute production of radiocarbon and the long-term trend of atmospheric radiocarbon. Radiocarbon 43(2B): 743-9.

Jenkins GM, Watts DG. 1969. Spectral Analysis and Its
Applications. London: Holden-Day. 525 p.

Kocharov GE, Arslanov KA, Dergachev VA, Tleugaliev SK, Chernov SB. 1977. Cyclic activity of the sun and the radiocarbon content in tree rings. Soviet Astronomical Letters 3(5):257-8.

Kocharov GE, Vasilev VA, Dergachjev VA, Ostryakov VM. 1983. An 8000-year sequence of galactic cosmicray fluctuations. Soviet Astronomical Letters 9(2): $110-2$.

Oeschger H, Siegenthaler U, Schotterer U, Gugelmann A. 1974. A box diffusion model to study the carbon dioxide exchange in nature. Tellus 27(2):168-92.

Siegenthaler U, Heimann M, Oeschger H. $1980 .{ }^{14} \mathrm{C}$ variations caused by changes in the global carbon cycle. Radiocarbon 22(2):177-91.

Stuiver M, Braziunas TF, Becker B, Kromer B. 1991. Climatic, solar, oceanic, and geomagnetic influences on Late Glacial and Holocene atmospheric ${ }^{14} \mathrm{C} /{ }^{12} \mathrm{C}$ change. Quaternary Research 35:1-24.

Stuiver M, Quay P. 1980. Patterns of atmospheric ${ }^{14} \mathrm{C}$ changes. Radiocarbon 22(2):166-76.

Stuiver M, Reimer PJ, Bard E, Burr GS, Hughen KA, Kromer B, McCormac G, van der Plicht J, Spurk M. 1998. IntCal98 radiocarbon age calibration, $24,000-0$ cal BP. Radiocarbon 40(3):1041-83.

Suess HE. 1955. Radiocarbon content in modern wood. Science 122:415-7. 